PDES, SUBMANIFOLDS AND

AFFINE DIFFERENTIAL GEOMETRY

BANACH CENTER PUBLICATIONS, VOLUME 69

INSTITUTE OF MATHEMATICS

POLISH ACADEMY OF SCIENCES

WARSZAWA 2005

\title{
A VARIATIONAL SOLUTION OF THE A. D. ALEKSANDROV PROBLEM OF EXISTENCE OF A CONVEX POLYTOPE WITH PRESCRIBED GAUSS CURVATURE
}

\author{
VLADIMIR OLIKER \\ Department of Mathematics and Computer Science, Emory University \\ Atlanta, GA 30322, U.S.A. \\ E-mail: oliker@mathcs.emory.edu
}

\begin{abstract}
In his book on convex polytopes [2] A. D. Aleksandrov raised a general question of finding variational formulations and solutions to geometric problems of existence of convex polytopes in $\mathbb{R}^{n+1}, n \geq 2$, with prescribed geometric data. Examples of such problems for closed convex polytopes for which variational solutions are known are the celebrated Minkowski problem [2] and the Gauss curvature problem [20]. In this paper we give a simple variational proof of existence for the A. D. Aleksandrov problem $[1,2]$ in which the hypersurface in question is a polyhedral convex graph over the entire $\mathbb{R}^{n}$, has a prescribed asymptotic cone at infinity, and whose integral Gauss-Kronecker curvature has prescribed values at the vertices. The functional that we use is motivated by the functional arising in the dual problem in the Monge-Kantorovich optimal mass transfer theory considered by W. Gangbo [13] and L. Caffarelli [11]. The presented treatment of the Aleksandrov problem is self-contained and independent of the Monge-Kantorovich theory.
\end{abstract}

1. Introduction. We recall first the original problem of A. D. Aleksandrov [1, 2]. Let $S^{n}$ be the unit sphere in $\mathbb{R}^{n+1}, n \geq 2$, with the center $O$ at the origin of a Cartesian coordinate system $(x, z) \equiv\left(x_{1}, \ldots, x_{n}, z\right)$. Denote by $\alpha$ the hyperplane $z=0$. Let $F$ be a noncompact, complete and convex (not necessarily smooth) hypersurface in $\mathbb{R}^{n+1}$. It will be convenient to assume that the direction of the axis $z$ is such that a ray parallel to the $z$-axis is contained inside the convex body bounded by $F$. This can always be achieved by performing (if necessary) a rotation of coordinate axes. For a point $x \in \alpha$, which is a projection of some point $X \in F$, we let $N(x)$ denote the set of outward

2000 Mathematics Subject Classification: Primary 53C45, 49K20; Secondary 35J65.

The research of the author was partially supported by a grant from Emory University Research Committee.

The paper is in final form and no version of it will be published elsewhere. 
normals to all supporting hyperplanes to $F$ at $X$. Thus, we have a generalized Gauss map $x \rightarrow X(x) \rightarrow N(x) \in S^{n}$. For a set $\omega \subset \alpha$ let $\tilde{\omega}$ be the subset of $\omega$ each point of which is a projection of some point of $F$. Put $\nu_{F}(\omega)=\bigcup_{x \in \tilde{\omega}} N(x)$. If $\omega$ is such that $\tilde{\omega}=\emptyset$ then we put $\nu_{F}(\omega)=\emptyset$. The set $\nu_{F}(\omega)$ is Lebesgue measurable for any Borel $\omega \subset \alpha$ (see, for example, [5], ch 1). Let $\left|\nu_{F}(\omega)\right|$ be the $n$-volume of $\nu_{F}(\omega)$ on $S^{n}$. The function $\left|\nu_{F}\right|$ on Borel subsets of $\alpha$ is a nonnegative and completely additive measure. It is called the integral Gauss (三 Gauss-Kronecker) curvature.

Let $B_{F}$ be the solid convex body bounded by $F$. Pick a point $Q \in B_{F}$ and consider all rays emanating from $Q$ and contained in $B_{F}$. The closure of this set of rays forms a closed convex cone. Its boundary $K_{F}$ is called the asymptotic cone of $F$. For a noncompact complete convex $F$ such $K_{F}$ always exists and is unique up to a translation in $\mathbb{R}^{n+1}$. It is well known (and not difficult to see) that $\nu_{K_{F}}(\alpha)=\overline{\nu_{F}(\alpha)}$.

In 1942 A. D. Aleksandrov proved the following

TheOREM 1.1. Let $K$ be a convex cone in $\mathbb{R}^{n+1}$ such that any straight line parallel to the $z$-axis either does not intersect $K$ or its intersection with the convex body bounded by $K$ is a complete ray. Then, a given function $\mu$ on Borel subsets of $\alpha$ is the integral Gauss curvature of a complete convex hypersurface $F$ in $\mathbb{R}^{n+1}$ with the asymptotic cone $K$ if and only if: (1) $\mu$ is a nonnegative and completely additive measure on Borel subsets of $\alpha$, (2) for any point $x \in \alpha, \mu(x)<\sigma_{n} / 2$, where $\sigma_{n}$ is the $n$-volume of $S^{n}$, (3) $\mu(\alpha)=\left|\nu_{K}(\alpha)\right|$. Furthermore, such $\mu$ and $K$ define $F$ uniquely up to a translation in the direction of the $z$-axis.

Aleksandrov's proof of this theorem is divided into two steps. In step one he solves the problem in the "discrete" case when the measure $\mu$ is a finite sum of Dirac masses, and the cone $K$ is a polyhedral convex cone with a one-to-one projection on the hyperplane $\alpha$. In this case the solution is a complete convex polytope, whose existence is proved with the use of the so-called "mapping lemma" of Aleksandrov; see, for instance, [2] or $[22], \S 2$. Step two is an approximation procedure. First, the originally given measure $\mu$ is approximated by a sequence of finite sums of Dirac masses and the cone $K$ is approximated by convex polyhedral cones. This gives a sequence of convex polytopes $P_{k}$ solving the corresponding discrete problems. Then it is shown that the set of convex piece-wise linear functions whose graphs are $P_{k}$ is compact in $C(\alpha)$ and therefore one can extract a subsequence convergent to a complete convex hypersurface $F$. The integral Gauss curvatures of $P_{k}$ are weakly continuous and, consequently, $\left|\nu_{P_{k}}\right| \rightarrow\left|\nu_{F}\right|=\mu$.

In [2], p. 296, after describing a variational solution to the Minkowski problem for polytopes, A. D. Aleksandrov raises the general question of finding variational proofs of other existence theorems for convex polytopes. The purpose of this note is to give such a variational proof of Aleksandrov's theorem in the case of convex polytopes with nondegenerate convex asymptotic cones ${ }^{1}$. In fact, we prove a more general result in which the integral Gauss curvature is constructed with an arbitrary given nonnegative integrable weight function. By a different method the Aleksandrov problem, in such generalized form, was considered previously by I. J. Bakelman [4], [5], ch. 5, and A. V. Pogorelov [21].

\footnotetext{
${ }^{1}$ An asymptotic cone of a complete convex hypersurface is nondegenerate if it projects univalently onto the entire hyperplane $\alpha$.
} 
Our main result, Theorem 2.3 , is presented in section 2 where we construct and investigate a minimization problem for a functional very naturally connected with this geometric problem. Our functional is similar to the one arising in the dual problem associated with the Monge-Kantorovich optimal mass transfer problem; see, especially, L. Caffarelli [11] and W. Gangbo [13] and related papers by Y. Brenier [6, 7], W. Gangbo and R. J. McCann $[14,15]$, L. Caffarelli $[9,8,10]$ as well as our recent papers $[17,16]$ and the paper by X.-J. Wang [24] dealing with problems in geometrical optics. However, the variational treatment of Aleksandrov's problem presented here is self-contained and does not depend on the Monge-Kantorovich theory.

In section 3 we collect several remarks complementing our main result, including a remark showing the connection between the problem of Aleksandrov and the problem of G. Monge from Monge-Kantorovich optimal mass transfer theory.

Finally, we note that in [19], R. J. McCann proved a result which implies, in particular, Aleksandrov's theorem in the case when the measure $\mu$ vanishes on Borel subsets of Hausdorff dimension $n-1$. Thus, formally, it does not cover the case of convex polytopes considered in this paper. However, in essence, the proof for this case can be extracted from the proof in [19]. Still, the treatment below is more direct and elementary and it is carried out in a geometric setting. Our approach should be useful in other geometric problems.

2. The variational problem. Let $W_{+}$denote the set of convex ${ }^{2}$ functions defined on the entire hyperplane $\alpha$. For a function $u \in W_{+}$we denote by $F_{u}$ the graph of $u$. At any point $X^{0}=\left(x^{0}, u\left(x^{0}\right)\right) \in F_{u}$ there exists at least one hyperplane supporting to $F_{u}$ at $X^{0}$. Let

$$
z-u\left(x^{0}\right)=\sum_{i=1}^{n} p_{i}^{0}\left(x_{i}-x_{i}^{0}\right)
$$

be the equation of a supporting hyperplane at $X^{0}$ with normal $\left(p^{0},-1\right)=\left(\left(p_{1}^{0}, \ldots, p_{n}^{0}\right)\right.$, $-1)$. Clearly,

$$
u(x) \geq\left\langle p^{0}, x\right\rangle-\left\langle p^{0}, x^{0}\right\rangle+u\left(x^{0}\right) \quad \forall x \in \alpha .
$$

Let $\chi_{u}\left(x^{0}\right)=\bigcup\left\{p^{0}\right\}$, where $p^{0}$ are vectors in $\mathbb{R}^{n}(\equiv \alpha)$ defined by hyperplanes (1) supporting $F_{u}$ at $X^{0}$ and the union is taken over all supporting hyperplanes at $X^{0}$. The set $\chi_{u}\left(x^{0}\right)$ is called the normal image of $x^{0}$. For any subset $\omega \subset \alpha$ we put

$$
\chi_{u}(\omega)=\bigcup_{x \in \omega} \chi_{u}(x)
$$

and call it the normal image of $\omega$. It is known (see, for instance, Bakelman [5], p. 116) that for any Borel set $\omega \subset \alpha$ the set $\chi_{u}(\omega)$ is Lebesgue measurable. For a function $u \in W_{+}(\alpha)$ a point $(x, u(x))$ on its graph $F_{u}$ is called a true vertex if $\left|\chi_{F_{u}}(x)\right|>0$, where $|\cdot|$ denotes the $n$-dimensional volume on $\alpha$.

For a given finite set of points $\mathcal{A}=\left\{a^{1}, \ldots, a^{k}\right\} \subset \alpha, k \geq 1$, we denote by $W_{+}(\mathcal{A})$ the subset of $W_{+}$consisting of piece-wise linear functions whose graphs may have vertices only

\footnotetext{
${ }^{2}$ We deal here only with convex functions but all of our considerations apply also, almost verbatim, to concave functions.
} 
on straight lines perpendicular to $\alpha$ and passing through the points $a^{1}, \ldots, a^{k}$. Denote by $\bar{\Omega}$ the convex hull of the points $a^{1}, \ldots, a^{k}$.

Lemma 2.1. Let $u \in W_{+}(\mathcal{A}), F_{u}^{\prime}=\{(x, u(x)), x \in \partial \Omega\}$ and $\bar{x}$ an arbitrary point in $\alpha \backslash \bar{\Omega}$. For any supporting hyperplane $P$ to $F_{u}$ at $\bar{X}=(\bar{x}, u(\bar{x}))$ the contact set $P_{\text {con }}=P \cap F_{u}$ has a non-empty intersection with $F_{u}^{\prime}$. Moreover, the point $\bar{X}$ can be connected by a straight line segment lying in $P_{\text {con }}$ with at least one of the vertices $\left(a^{i}, u\left(a^{i}\right)\right)$.

Proof. Obviously, if $P_{\text {con }}=\{\bar{X}\}$ then the vector $p$ defined by the normal $(p,-1)$ to supporting hyperplane $P$ is an interior point of $\chi_{u}(\bar{x})$. Then $\bar{X}$ is a true vertex of $F_{u}$, which contradicts our choice of $\bar{X}$. Since $P_{c o n}$ is convex, there exists a straight line segment connecting $\bar{X}$ with any boundary point in $\partial P_{\text {con }}$.

On the other hand, if $\partial P_{\text {con }} \cap F_{u}^{\prime}=\emptyset$ then there must exist a true vertex in $\partial P_{c o n}$ which is, again, impossible. Hence, $\bar{X}$ can be connected to some point on $F_{u}^{\prime}$ by a linear segment. In fact, by convexity of $P_{\text {con }} \bar{X}$ can be connected by a linear segment to at least one of the vertices $\left(a^{i}, u\left(a^{i}\right)\right)$.

This lemma and the fact that $u$ is defined over the entire $\alpha$ imply that $F_{u}$ cannot have vertical supporting hyperplanes. Furthermore, it also implies that the normal image $\bar{T}_{u} \equiv \chi_{u}(\alpha)$ is compact on $\alpha$. Moreover, it is not difficult to see that the asymptotic cone $K_{u}$ of $F_{u}$ is convex and $\chi_{K_{u}}(\alpha)=\bar{T}_{u}$. Hence, $\bar{T}_{u}$ is also convex.

The epigraph of $u$ is an infinite complete convex body in $\mathbb{R}^{n+1}$ whose boundary is the convex polytope $F_{u}$. For each $p \in \bar{T}_{u}$ there is a supporting hyperplane to $F_{u}$ with the equation $z=\langle x, p\rangle-v(p)$. Then, since the epigraph of $u$ is the intersection of half-spaces defined by its supporting hyperplanes, we have

$$
u(x)=\sup _{p \in \bar{T}_{u}}[\langle x, p\rangle-v(p)] \quad \forall x \in \alpha
$$

and for each $p \in \bar{T}_{u}$ there exists at least one $a^{i} \in \mathcal{A}$ such that

$$
u\left(a^{i}\right)=\left\langle a^{i}, p\right\rangle-v(p) .
$$

By (3) we have

$$
v(p) \geq\langle x, p\rangle-u(x) \quad \forall p \in \bar{T}_{u} \text { and } \forall x \in \alpha
$$

and the equality is achieved at $x$ where the hyperplane $z=\langle x, p\rangle-v(p)$ is supporting to $F_{u}$. In other words,

$$
v(p)=\sup _{x \in \alpha}[\langle x, p\rangle-u(x)], p \in \bar{T}_{u},
$$

which also means that $v$ is a convex function on $\bar{T}_{u}$. The function $v$ is the LegendreFenchel (LF) transform of $u$ which is the generalization of the usual Legendre transform to non-smooth convex functions. We will also use the notation $u^{*}$ to denote the LF transform of $u$. Note that $\left(u^{*}\right)^{*}=u$; see, for example, R. Schneider [23], p. 36.

Let $\bar{T}$ be a compact convex set on $\alpha$ with interior points (in topology of $\alpha$ ). Let $p \in \bar{T}$ and

$$
L_{+}(p)=\left\{(x, z) \in \mathbb{R}^{n+1} \mid z \geq\langle x, p\rangle\right\}
$$

a half-space defined by the hyperplane $z=\langle x, p\rangle$ with the normal $(p,-1)$. Put

$$
K=\partial\left(\bigcap_{p \in \bar{T}} L_{+}(p)\right) \text {. }
$$


Obviously, $K$ is a convex cone with vertex at $O$ defined over the entire hyperplane $\alpha$. If the boundary $\partial \bar{T}$ is a convex polytope then $K$ is an infinite convex polytope.

For the rest of this section we consider only the case when the boundary of $\bar{T}$ is a convex polytope.

Denote by $W_{+}(\mathcal{A}, K)$ the subset of functions in $W_{+}(\mathcal{A})$ whose asymptotic cone is a parallel translate of $K$. This set is not empty as, in particular, the cone $K$ translated to any of the points $a^{i}$ is the graph of a function in $W_{+}(\mathcal{A}, K)$. The set of functions which are LF transforms of functions from $W_{+}(\mathcal{A}, K)$ we denote by $W_{+}^{*}(\mathcal{A}, K)$. Finally, put

$$
A d m(\alpha, \bar{T})=\left\{(\xi, \tau) \in W_{+}(\mathcal{A}, K) \times W_{+}^{*}(\mathcal{A}, K) \mid \xi(x)+\tau(p) \geq\langle x, p\rangle \forall(x, p) \in \alpha \times \bar{T}\right\} .
$$

It follows from (3) (or (5)) that $\operatorname{Adm}(\alpha, \bar{T})$ is not empty.

Lemma 2.2. Let $(\xi, \tau) \in \operatorname{Adm}(\alpha, \bar{T})$. Then $\xi$ and $\tau$ are both uniformly Lipschitz continuous on $\alpha$ and $\bar{T}$, respectively.

Proof. Since any $\xi \in W_{+}(\mathcal{A}, K)$ has an asymptotic cone which is a translate of $K$, it is clear that $\xi$ is uniformly Lipschitz with a Lipschitz constant not exceeding $\max _{\bar{T}}|p|$.

An alternative analytic proof can be given as follows. Let $x^{1}, x^{2} \in \alpha$. Denote by $\xi^{*}$ the LF of $\xi$. We have for some $\bar{p} \in \bar{T} \xi\left(x^{2}\right)=\left\langle x^{2}, \bar{p}\right\rangle-\xi^{*}(\bar{p})$ and $\xi\left(x^{1}\right) \geq\left\langle x^{1}, \bar{p}\right\rangle-\xi^{*}(\bar{p})$. Then

$$
\xi\left(x^{2}\right)-\xi\left(x^{1}\right) \leq\left\langle x^{2}, \bar{p}\right\rangle-\xi^{*}(\bar{p})-\left\langle x^{1}, \bar{p}\right\rangle+\xi^{*}(\bar{p}) \leq \max _{\bar{T}}|p|\left|x^{2}-x^{1}\right| .
$$

Similarly, we obtain the inequality

$$
\xi\left(x^{2}\right)-\xi\left(x^{1}\right) \geq-\max _{\bar{T}}|p|\left|x^{2}-x^{1}\right|
$$

The uniform Lipschitz continuity of $\tau$ is shown in the same way, and, in fact, for any $p^{1}, p^{2} \in \bar{T}$ we have

$$
\left|\tau\left(p^{2}\right)-\tau\left(p^{1}\right)\right| \leq \max _{i=1, \ldots, k}\left|a^{i}\right|\left|p^{2}-p^{1}\right|
$$

THEOREM 2.3. Let $\bar{T}$ be a compact convex set on $\alpha$ with interior points and boundary $\partial \bar{T}$ which is a convex polytope. Let $L$ be a nonnegative integrable function on $T$ and $g_{1}, \ldots, g_{k}$ nonnegative numbers such that

Put

$$
\sum_{i=1}^{k} g_{i}=\int_{T} L(p) d p \neq 0
$$

$$
\mathcal{F}(\xi, \tau)=\sum_{i=1}^{k} g_{i} \xi\left(a^{i}\right)+\int_{T} \tau(p) L(p) d p, \quad(\xi, \tau) \in \operatorname{Adm}(\alpha, \bar{T}) .
$$

Then there exists a convex polytope which is the graph of a function $u \in W_{+}(\mathcal{A}, K)$, where $K$ is the convex cone defined by $\bar{T}$ as in (6), such that for $u$ and its LF transform $v \in W_{+}^{*}(\mathcal{A}, K)$ we have

$$
\mathcal{F}(u, v)=\inf _{\operatorname{Adm}(\alpha, \bar{T})} \mathcal{F}(\xi, \tau) .
$$

For any u satisfying (10) we have

$$
\int_{\chi_{u}\left(a^{i}\right)} L(p) d p=g_{i}, i=1,2, \ldots, k .
$$

Furthermore, if $L>0$ in $T$ then $u$ is unique up to an additive constant. 
Proof. First note that for any constant $C$ the function $\xi+C$ remains in $W_{+}(\mathcal{A}, K)$ and for its LF transform we have $(\xi+C)^{*}=\xi^{*}-C$. By $(8) \mathcal{F}(\xi+C, \tau-C)=\mathcal{F}(\xi, \tau)$ and we may restrict the search for minimizers to $\xi$ in $W_{+}(\mathcal{A}, K)$ such that $\xi\left(a^{1}\right)=0$. Let $W_{+, 0}(\mathcal{A}, K)$ be the subset of functions in $W_{+}(\mathcal{A}, K)$ satisfying this additional condition. By lemma 2.2 the set $A d m_{0}(\alpha, T)$ constructed with the use of $W_{+, 0}(\mathcal{A}, K)$ is compact in $C(\alpha) \times C(\bar{T})$. Since the functional $\mathcal{F}$ is continuous on $C(\alpha) \times C(\bar{T})$, there exists a pair $(u, v) \in A d m_{0}(\alpha, T)$ satisfying (10).

Next we observe that the function $v$ in (10) can be assumed to be the LF transform of $u$. Indeed, by definition of $\operatorname{Adm}(\alpha, T) v(p) \geq\langle x, p\rangle-u(x) \forall x \in \alpha$ and then $v(p) \geq$ $\sup _{\alpha}[\langle x, p\rangle-u(x)]=u^{*}(p)$. But then $\mathcal{F}(u, v) \geq \mathcal{F}\left(u, u^{*}\right)$. This also implies that when minimizing $\mathcal{F}$ it suffices to look for minimizers among pairs which are LF transforms of each other; cf. Gangbo-McCann [14].

Now we prove (11). First we prove it under the following assumption.

(A) The function $u$ in the minimizing pair $(u, v)$ is such that $\left|\chi_{u}\left(a^{1}\right)\right|>0$.

Consider the following variation of $u$. Pick $\gamma>0$ and consider the convex polytope $F_{u_{\gamma}}$ defined by vertices $\left(a^{1}, u_{\gamma}\left(a^{1}\right)=u\left(a^{1}\right)+\gamma\right),\left(a^{i}, u\left(a^{i}\right)\right), i>1$, and the cone $K$ translated so that its vertex is positioned at any one of the points $\left(a^{i}, u\left(a^{i}\right)\right), i>1$. Such a polytope is the boundary of the convex hull of the (translated) cone $K$, vertices $\left(a^{i}, u\left(a^{i}\right)\right), i>1$, and vertex $\left(a^{1}, u_{\gamma}\left(a^{1}\right)\right)$. Denote this polytope by $F_{u_{\gamma}}$. Since $\left|\chi_{u}\left(a^{1}\right)\right|>0$, we can pick $\gamma$ so that $\left|\chi_{u_{\gamma}}\left(a^{1}\right)\right|>0$. The vertices $\left(a^{i}, u\left(a^{i}\right)\right)$ on $F_{z}$ can only be of the following two types: (a) $\left|\chi_{u}\left(a^{i}\right)\right|>0$ and (b) $\left|\chi_{u}\left(a^{i}\right)\right|=0$. We can choose $\gamma$ sufficiently small so that the vertices of type (a) will remain of the same type on $F_{u_{\gamma}}$. The vertices of type (b) may become of type (a) on $F_{u_{\gamma}}$.

It is easy to see that $\chi_{u_{\gamma}}\left(a^{1}\right) \subset \chi_{u}\left(a^{1}\right)$, while $\chi_{u}\left(a^{i}\right) \subseteq \chi_{u_{\gamma}}\left(a^{i}\right)$ for $i>1$. Put $\kappa_{i j}=\chi_{u_{\gamma}}\left(a^{i}\right) \cap \chi_{u}\left(a^{j}\right)$. Then

$$
\begin{gathered}
\chi_{u_{\gamma}}\left(a^{1}\right)=\chi_{u}\left(a^{1}\right) \backslash\left(\bigcup_{i>1} \kappa_{i 1}\right), \\
\chi_{u_{\gamma}}\left(a^{i}\right)=\chi_{u}\left(a^{i}\right) \cup \kappa_{i 1}, \quad \text { for } i \neq 1 .
\end{gathered}
$$

Next, we note that for $i \neq 1$ and $p \in \chi_{u}\left(a^{i}\right)$ the LT transform of $u_{\gamma}$ is $v_{\gamma}(p)=\sup _{x \in \alpha}\left[\langle x, p\rangle-u_{\gamma}(x)\right]=\left\langle a^{i}, p\right\rangle-u_{\gamma}\left(a^{i}\right)=\left\langle a^{i}, p\right\rangle-u\left(a^{i}\right)=\sup _{x \in \alpha}[\langle x, p\rangle-u(x)]=v(p)$. Therefore,

$$
\begin{aligned}
\int_{T}\left[v_{\gamma}(p)-v(p)\right] L(p) d p & =\int_{\chi_{u}\left(a^{1}\right)}\left[v_{\gamma}(p)-v(p)\right] L(p) d p+\sum_{i>1} \int_{\chi_{u}\left(a^{i}\right)}\left[v_{\gamma}(p)-v(p)\right] L(p) d p \\
& =\int_{\chi_{u_{\gamma}}\left(a^{1}\right)}\left[v_{\gamma}(p)-v(p)\right] L(p) d p+\sum_{i>1} \int_{\kappa_{i 1}}\left[v_{\gamma}(p)-v(p)\right] L(p) d p \\
& \leq-\gamma \int_{\chi_{u_{\gamma}}\left(a^{1}\right)} L(p) d p
\end{aligned}
$$

where we made use of the inequalities: 


$$
\begin{aligned}
& v_{\gamma}(p)-v(p)=\left\langle a^{1}, p\right\rangle-u_{\gamma}\left(a^{1}\right)-\sup _{x \in \alpha}[\langle x, p\rangle-u(x)] \leq-\gamma \quad \forall p \in \chi_{u}\left(a^{1}\right), \\
& v_{\gamma}(p)-v(p)=\left\langle a^{i}, p\right\rangle-u_{\gamma}\left(a^{i}\right)-\sup _{x \in \alpha}[\langle x, p\rangle-u(x)] \leq 0 \quad \forall p \in \kappa_{i 1}, i>1 .
\end{aligned}
$$

Next, we note that for $i>1$

$$
\int_{\kappa_{i 1}} L(p) d p=O(\gamma)
$$

This can be seen as follows. Denote by $C$ the polyhedral cone formed by facets of $F_{u}$ adjacent to $\left(a^{1}, u\left(a^{1}\right)\right)$. Let $C^{*}$ be the polyhedral cone dual to $C$ with vertex $\left(a^{1}, u\left(a^{1}\right)\right)$. Translate the coordinate system in $\mathbb{R}^{n+1}$ so that the vertex $\left(a^{1}, u\left(a^{1}\right)\right)$ becomes the origin. Then, $\chi_{u}\left(a^{1}\right)$ is a compact convex set on the hyperplane $z=-1$ whose boundary is a convex polytope. Using the duality between $C$ and $C^{*}$, it can be seen that when the vertex $\left(a^{1}, u\left(a^{1}\right)\right)$ is moved in the $z$-direction by a distance $\gamma$ the $(n-1)$-dimensional faces of $\chi_{u}\left(a^{1}\right)$ move parallel to themselves by a distance proportional to $\gamma$.

Thus, (12), (14) and (10) imply

$$
0 \leq \mathcal{F}\left(u_{\gamma}, v_{\gamma}\right)-\mathcal{F}(u, v) \leq \gamma\left[g_{1}-\int_{\chi_{u}\left(a^{1}\right)} L(p) d p\right]+o(\gamma)
$$

Replacing $\gamma$ by $-\gamma$ and using the same arguments we obtain the reverse inequality. This proves (11).

Now we remove the assumption (A) made at the beginning of this proof. First, we note that if $\left|\chi_{u}\left(a^{1}\right)\right|=0$ then the same arguments that were used to establish (12) and (14) show that

which implies that

$$
\int_{T}\left[v_{\gamma}(p)-v(p)\right] L(p) d p=o(\gamma)
$$

$$
g_{1}=\int_{\chi_{u}\left(a^{1}\right)} L(p) d p=0
$$

Finally, we note that an arbitrary variation of $u$ is a linear superposition of displacements of vertices $\left(a^{1}, u\left(a^{1}\right)\right), \ldots,\left(a^{k}, u\left(a^{k}\right)\right)$ by sufficiently small numbers $\gamma_{1}, \ldots, \gamma_{k}$, and this implies (11) in the general case.

Uniqueness of a solution up to an additive constant is well known; see, [2], ch. IX, or [5], p. 211.

\section{Remarks}

REMARK 1. Using Theorem 2.3 one can obtain a similar result for general convex hypersurfaces.

TheOREM 3.1. Let $\bar{T}$ be a compact convex set in $\alpha$ with interior points (in the topology of $\alpha$ ) and $L$ a nonnegative integrable function on $T$ such that

$$
\int_{T} L(p) d p \neq 0
$$

Let $\mu$ be a nonnegative and completely additive function on Borel subsets of $\alpha$ such that

$$
\mu(\alpha)=\int_{T} L(p) d p
$$

Then there exists a complete convex hypersurface $F$ defined over $\alpha$ such that its asymptotic 
cone is the cone $K$ defined by the set $\bar{T}$ as in (6) and for each Borel set $\omega \subset \alpha$

$$
\mu(\omega)=\int_{\chi_{F}(\omega)} L(p) d p,
$$

where $\chi_{F}(\omega)$ is the normal image of $\omega$. Such $F$ is unique up to a parallel translation in the direction of the z-axis.

The proof of this theorem is obtained by the same approximation procedure as the proof of Aleksandrov's theorem described in the introduction, except that instead of the mapping lemma one can use Theorem 2.3 to construct polyhedral solutions to the corresponding discrete problems. The details of this procedure are well known and we refer the reader to the books $[2,5]$ and further references there.

REMARK 2. The sufficiency part in Aleksandrov's theorem in case when the cone $K$ is nondegenerate is obtained if one takes in the previous remark

$$
L(p)=\frac{1}{\left(1+p^{2}\right)^{(n+1) / 2}} .
$$

Indeed, the generalized Gauss image of the cone $K$ is contained in a hemi-sphere $S_{-}^{n}$ of $S^{n}$ with the pole corresponding to the negative direction of the $z$-axis. Parametrize $S_{-}^{n}$ by the points $(p,-1), p \in \alpha$, on the hyperplane $H: z=-1$ using radial projection from $O$ of $S_{-}^{n}$ onto $H$. Then the volume element of $S_{-}^{n}$ is given by $\left(1+p^{2}\right)^{-(n+1) / 2} d p$. Thus, the right-hand side of (17) in this case is the integral Gauss curvature.

Furthermore, note that the condition (2) in Aleksandrov's theorem is automatically satisfied in our case because the cone $K$ is assumed to be nondegenerate and condition (16) holds. Indeed, the convex cone $K$ determines the set $\bar{T} \subset \alpha$ uniquely and since $K$ is nondegenerate, it does not have vertical supporting hyperplanes. Hence $\bar{T}$ is compact (and convex). Then (16) implies the condition (2).

REMARK 3 (Connection between the Aleksandrov and Monge problems). The problem discussed in the previous section can also be considered from a different point of view. Let $\mathcal{A}, g_{1}, \ldots, g_{k}, \bar{T}$ and $L$ be as in Theorem 2.3. Consider the class of maps $\Theta$, possibly multivalued, such that each $\theta \in \Theta$ has the following properties:

1. $\theta: \alpha \rightarrow \bar{T}$ and the image of any Borel set on $\alpha$ is Lebesgue measurable on $\alpha$,

2. The set $E \subset \bar{T}$ where $\theta^{-1}$ is multivalued has measure zero,

3. For any function $h \in C(\alpha)$

$$
\int_{T} h\left(\theta^{-1}(p)\right) L(p) d p=\sum_{i=1}^{k} h\left(a^{i}\right) g_{i} .
$$

Note that because $\theta^{-1}$ may be multivalued the integrand on the left of (18) may not be defined at all $p \in \bar{T}$. However, because of property 2 this may happen only on a set of measure zero. Hence, the left hand side of (18) is well defined. The property 3 is referred to as the "measure preserving" property of $\theta$.

The set $\Theta \neq \emptyset$. For example, if the condition (8) is satisfied then the normal map $\chi_{u}: \alpha \rightarrow \bar{T}$, where $(u, v)$ is a minimizing pair in Theorem 2.3, is in $\Theta$. More generally, assuming again that (8) holds we partition $\bar{T}$ into closed subsets $\bar{E}_{1}, \ldots, \bar{E}_{k}$ such that 
$\left|\partial E_{i}\right|=0, E_{i} \bigcap E_{j}=\emptyset$ when $i \neq j, \bar{T}=\bigcup_{i=1}^{k} \bar{E}_{i}$ and

$$
\int_{E_{i}} L(p) d p=g_{i}, i=1, \ldots, k .
$$

Consider now a map $\theta: \alpha \rightarrow \bar{T}$ such that for each $i \theta\left(a^{i}\right)=E_{i}$ and for any $x \in \alpha, x \neq a^{i}$, $\theta(x) \in \bigcup_{i=1}^{k} \partial E_{i}$. Clearly, $\theta \in \Theta$.

Here we show that the variational solution in Theorem 2.3 is tightly connected with the Monge problem in the Monge-Kantorovich optimal transport theory; see, for instance, L. V. Kantorovich and G. P. Akilov [18], Y. Brenier [7], C. Evans [12], L. Ambrosio [3] and other references there. In our setting the Monge prbolem can be formulated as follows. Let $\mathcal{A}, g_{1}, \ldots, g_{k}, \bar{T}$ and $L$ be as in Theorem 2.3 and $\Theta$ the set of maps as above. The problem consists in finding a map $\theta_{0} \in \Theta$ such that

$$
\sup _{\theta \in \Theta} \int_{T}\left\langle\theta^{-1}(p), p\right\rangle L(p) d p=\int_{T}\left\langle\theta_{0}^{-1}(p), p\right\rangle L(p) d p .
$$

The solution to this problem follows essentially from Theorem 2.3. The arguments below are motivated by the arguments in W. Gangbo [13].

Consider the set $A d m_{0}(\alpha, \bar{T})$ as in the proof of Theorem 2.3. For a pair $(\xi, \tau) \in$ $A d m_{0}(\alpha, \bar{T})$ we have

$$
\xi(x)+\tau(p) \geq\langle x, p\rangle \forall x \in \alpha, \forall p \in \bar{T} .
$$

Then, for any $\theta \in \Theta$ we have

$$
\int_{T} \xi\left(\theta^{-1}(p)\right) L(p) d p+\int_{T} \tau(p) L(p) d p \geq \int_{T}\left\langle\theta^{-1}(p), p\right\rangle L(p) d p
$$

and using the measure-preserving property of $\theta$ we get

$$
\sum_{i=1}^{k} \xi\left(a^{i}\right) g_{i}+\int_{T} \tau(p) L(p) d p \geq \int_{T}\left\langle\theta^{-1}(p), p\right\rangle L(p) d p=\sum_{i=1}^{k}\left\langle a^{i}, \int_{\theta\left(a^{i}\right)} p L(p) d p\right\rangle .
$$

Let $(u, v)$ be the minimizing pair of the functional on the left of $(22)$ whose existence is guaranteed by Theorem 2.3, and let $\chi_{u}$ be the corresponding normal map. Then for each $i=1, \ldots, k$ we have

$$
u\left(a^{i}\right)+v(p)=\left\langle a^{i}, p\right\rangle \forall p \in \chi_{u}\left(a^{i}\right) .
$$

Multiplying this equation for each $i$ by $L$, integrating over $\chi_{u}\left(a^{i}\right)$, and taking into account (11), we obtain

$$
\sum_{i=1}^{k} u\left(a^{i}\right) g_{i}+\int_{T} v(p) L(p) d p=\sum_{i=1}^{k}\left\langle a^{i}, \int_{\chi_{u}\left(a^{i}\right)} p L(p) d p\right\rangle .
$$

Thus, in (22) we have equality when $\xi=u, \tau=v$ and $\theta=\chi_{u}$ with $\theta_{0}=\chi_{u}$ being the solution of the Monge problem.

\section{References}

[1] A. D. Aleksandrov, Existence and uniqueness of a convex surface with a given integral curvature, DAN USSR 35 (1942), no. 5, 131-134. 
[2] A. D. Aleksandrov, Convex Polyhedra. GITTL, Moscow (in Russian), German transl.: Konvexe Polyeder, Akademie-Verlag, Berlin, 1958, 1950.

[3] L. Ambrosio, Lecture notes on optimal transport problems, CIME Series of Springer Lecture Notes, http://cvgmt.sns.it/papers/amb00a, pages 1-62, 2000.

[4] I. J. Bakelman, On the theory of Monge-Ampère equations, Vestnik Leningrad Univ. 1 (1958), 25-38.

[5] I. J. Bakelman, Convex Analysis and Nonlinear Geometric Elliptic Equations, SpringerVerlag, Berlin, 1994.

[6] Y. Brenier, Décomposition polaire et réarrangement monotone des champs de vecteurs, C. R. Acad. Sci. Paris 305 (1987), 805-808.

[7] Y. Brenier, Polar factorization and monotone rearrangement of vector-valued functions, Comm. in Pure and Applied Math. 44 (1991), 375-417.

[8] L. A. Caffarelli, Boundary regularity of maps with convex potentials, Comm. in Pure and Applied Math. 45 (1992), 1141-1151.

[9] L. A. Caffarelli, The regularity of mappings with convex potentials, J. of AMS 5 (1992), 99-104.

[10] L. A. Caffarelli, Allocation maps with general cost functions, Partial Differential Equations and Applications 177 (1996), 29-35.

[11] L. A. Caffarelli, Boundary regularity of maps with convex potentials. II, Ann. of Math. 144 (1996), 453-496.

[12] L. C. Evans, Partial differential equations and Monge-Kantorovich mass transfer, in: Current Developments in Mathematics, R. Bott et al. (eds.), International Press, Boston, 1997, 26-78.

[13] W. Gangbo, An elementary proof of the polar factorization of vector-valued functions, Arch. Rational Mech. Anal. 128 (1994), 381-399.

[14] W. Gangbo and R. J. McCann, Optimal maps in Monge's mass transport problem, C. R. Acad. Sci. Paris Sér. I Math. 321 (1995), 1653-1658.

[15] W. Gangbo and R. J. McCann, The geometry of optimal transportation, Acta Math. 177 (1996), 133-161.

[16] T. Glimm and V. Oliker, Optical design of single reflector systems and the MongeKantorovich mass transfer problem, J. of Math. Sciences 117 (2003), 4096-4108.

[17] T. Glimm and V. Oliker, Optical design of two-reflector systems, the Monge-Kantorovich mass transfer problem and Fermat's principle, Indiana Univ. Math. J. 53 (2004), 12551278.

[18] L. V. Kantorovich and G. P. Akilov, Functional Analysis, ch. VIII, §4, Nauka, Moscow, 1977, 2-nd revised edition (in Russian).

[19] R. J. McCann, Existence and uniqueness of monotone measure-preserving maps, Duke Math. J. 80 (1995), 309-323.

[20] V. I. Oliker, The problem of embedding $S^{n}$ into $R^{n+1}$ with prescribed Gauss curvature and its solution by variational methods, Transactions of the AMS 295 (1986), 291-303.

[21] A. V. Pogorelov, Extrinsic Geometry of Convex Surfaces, American Mathematical Society, Providence, R.I., 1973.

[22] A. V. Pogorelov, The Minkowski Multidimensional Problem, V. H. Winston, Washington, D.C., 1978.

[23] R. Schneider, Convex Bodies. The Brunn-Minkowski Theory, Cambridge Univ. Press, Cambridge, 1993.

[24] X. J. Wang, On design of a reflector antenna II, Calculus of Variations and PDE's 20 (2004), 329-341. 